\title{
An Open Design Microfabricated Nib-Like Nanoelectrospray Emitter Tip on a Conducting Silicon Substrate for the Application of the Ionization Voltage
}

\author{
Séverine Le Gac and Christian Rolando \\ Laboratoire de Chimie Organique et Macromoléculaire, Université des Sciences et Technologies de Lille, \\ Villeneuve d'Ascq Cedex, France \\ Steve Arscott* \\ Institut d'Electronique, de Microélectronique et de Nanotechnologie (IEMN), Cité Scientifique, Villeneuve \\ d'Ascq Cedex, France
}

\begin{abstract}
This paper describes a novel emitter tip having the shape of a nib and based on an open structure for nano-electrospray ionization mass spectrometry (nanoESI-MS). The nib structure is fabricated with standard lithography techniques using SU-8, an epoxy-based negative photoresist. The tip is comprised of a reservoir, a capillary slot and a point-like feature, and is fabricated on a silicon wafer. We present here a novel scheme for interfacing such nib tips to MS by applying the ionization voltage directly onto the semi-conductor support. The silicon support is in direct contact with the liquid to be analyzed at the reservoir and microchannel level, thus allowing easy use in ESI-MS. This scheme is especially advantageous for automated analysis as the manual step of positioning a metallic wire into the reservoir is avoided. In addition, the analysis performance was enhanced compared with the former scheme, as demonstrated by the tests of standard peptides (gramicidin S, Glu-fibrinopeptide B). The limit of detection was determined to be lower than $10^{-2} \mu \mathrm{M}$. Due to their enhanced performance, these microfabricated sources might be of great interest for analysis requiring very high sensitivity, such as proteomics analysis using nanoESI-MS. (J Am Soc Mass Spectrom 2006, 17, 75-80) (C) 2005 American Society for Mass Spectrometry
\end{abstract}

$\mathrm{T}$ The advent of the field of proteomics [1] has coincided with the explosion of (nano)-ESI-MS. Proteomics studies are carried out on small amounts of sample and, thereby, need highly sensitive analysis techniques. ESI-MS matches this sensitivity criterion [2]. It also enables species identification using their precise molecular weights and provides fragment sequences using the MS/MS mode [1, 3]. Therefore, ESI-MS is one of the best techniques for use in proteomics.

Nanospraying is usually performed using glass or fused-silica-based capillary tips [2], and the spray is obtained by applying the ionization voltage to the electrical conductive tip or through the sample solution. However, capillary sources present a number of

\footnotetext{
Published online December 15, 2005

Address reprint requests to Dr. C. Rolando, Université des Sciences et Technologies de Lille (Lille 1), Bâtiment C4, UMR CNRS 8009, Chimie Organique et Macromoléculaire, 59655 Villeneuve d'Ascq Cedex, France. E-mail: Christian.Rolando@univ-lille1.fr

* UMR CNRS 8520, Université des Sciences et Technologies de Lille, Villeneuve d'Ascq Cedex, France.
}

weaknesses: (1) a poorly controlled fabrication route resulting in a low reproducibility of the sources, (2) an irregular profile of the aperture after source opening, (3) easy clogging due to the small i.d., (4) signal suppression caused by air bubbles, and (5) deterioration of the conductive coating $[4,5]$ upon operation.

Different improvements regarding the quality of the emitter tip are proposed in the literature. Reproducible dimensions can be achieved using an enhanced fabrication route based on laser-micromachining [6, 7]. More stable coatings have also been reported [8-10]. Most of these improvements, however, do not solve the basic problems of clogging and the lack of reproducibility that affect the analysis conditions. Using microtechnology techniques, reproducible and ready-to-use high-quality nanospray tips can be produced in large batches. The tip geometry can be chosen to allow on-chip integration together with other microfluidic components, e.g., a chromatography column, and also to be compatible with the use of standard robots. Microfabrication of ESI emitter tips follows two main approaches: (1) a "needle-like" ap- 
proach or (2) a "channel-like" approach consisting of a sharpened interface at the outlet of a microchannel. "Needle-like" approaches were reported using silicon nitride [11], parylene [12], silicon [13-15], as well as polycarbonate [16] or polymethyl methacrylate (PMMA) [17]. The reported "channel-like" approaches include PMMA starshaped systems [18], polydimethyl siloxane (PDMS) devices having a triangular shape [19], devices based on a groove feature [20], machined point-like structures [21], polyimide-based triangular systems [22], and triangular parylene sheet-based devices [23].

Our approach is to design an emitter tip having the shape of a nib. Such a tip relies on a planar and open structure. Its topology and functioning mimic those of a fountain pen. Two series of prototypes have already been reported $[24,25]$. They work under nanoESI conditions (high voltage (HV) supply around $1 \mathrm{kV}$ ) provided that their dimensions are small enough. For the work reported here, we have only used nib tips having an $8 \mu \mathrm{m}$ width slot. A major improvement upon our previous work results from a direct application of the ionization voltage onto the supporting silicon $(\mathrm{Si})$ wafer instead of using an external metallic wire. This new scheme results in a lower current density, leading to gentler ionization conditions [24, 26, 27]. In addition, the nibs are expected to last longer, as HV application is made through an intrinsic part of the devices and not through a metal layer deposited on it, as is the case in pulled tips.

\section{Materials and Methods}

\section{Chemicals}

Standard peptides (gramicidin S, [Glu ${ }^{1}$-fibrinopeptide B) and solvents were purchased from Sigma-Aldrich (L'Isle d'Abeau, France). The solutions for the tests were prepared by diluting stock solutions using a $\mathrm{MeOH}$ : $\mathrm{H}_{2} \mathrm{O} 50 / 50,0.1 \%$ formic acid solvent to have a concentration in the range $10^{-2} \mu \mathrm{M}$ to $5 \mu \mathrm{M}$.

\section{Micronib Tip Layout and Fabrication}

The nib tips are composed of two main parts, as previously described [25, 28]: (1) a Si support and (2) a SU-8-based nib structure as illustrated in Figure 1. The capillary slot has a width $w$ of $8 \mu \mathrm{m}$, and the height $h$ of SU-8 resist at the tip of the nib is $\sim 35 \mu \mathrm{m}$. These two dimensions of the nib tip are critical for its functioning in ESI $[25,28]$. The microfabrication process of the micro-nib emitter tips has already been published in detail elsewhere [28].

\section{Mass Spectrometry Tests}

The nib emitter tips were tested on a LCQ Deca $\mathrm{XP}^{+}$ion trap mass spectrometer (Thermo Finnigan, San Jose, CA) as described previously [24, 25, 29]. The nib tip was introduced in the MS inlet using the standard holder, on which it was fixed using Teflon tape. For the experiments reported here, the mode of $\mathrm{HV}$ application was changed: HV was directly applied to the solution through the Si support wafer that is in contact with the metallic area of the holder. The total ion current (TIC) was recorded over 2 to 6 min for all experiments, and the mass spectra were plotted using Excalibur software (Thermo Finnigan, San Jose, CA) as an average over this acquisition time.

\section{Results and Discussion}

\section{Nib Structure and Functioning}

The principle of this nanoESI emitter tip is reminiscent of the functioning principle and the geometry of a calligraphic nib (see Figure 1). The idea of such an emitter tip has been recently validated through the fabrication and testing of two series of nib tips [24, 25, $28,30]$. The planar design of nib sources allows easy loading by droplet deposition that can be straightforwardly automated. Furthermore, its open structure limits the common problems of air bubbles and clogging. The reliability of these nibs was demonstrated for nanoESI applications [24, 25, 29]. Liquid flows to the nib tip by capillary action. Once the nib tip point is reached, liquid is ejected as a spray upon application of a high voltage. The observation of capillary action was therefore seen to depend on the dimensions of the slot feature and on the nature of the material. The height $h$ of the SU-8 at the nib tip must be greater than the capillary slot width $w$ to observe capillarity [31]. Indeed, for SU-8, which has neutral hydrophobic properties, the required capillary effect was not observed for nibs with a lesser height of resist $(h=15 \mu \mathrm{m}, w=20$ $\mu \mathrm{m})$ [28]. Another important point for ESI applications is the size of the slot that dictates the size of the generated droplets [2] as the size of the droplet is directly linked to the sensitivity of the analysis. The experiments reported here were carried out using nib tips having $8 \mu \mathrm{m}(w)$ and $35 \mu \mathrm{m}(h)$. These dimensions were appropriate for capillary action to occur in the slot and were fully compatible with nanoESI applications.

\section{Set-Up Used for the MS Tests}

We have previously tested these micro-nib emitter tips using a Pt wire that was inserted in the reservoir, allowing an electrical contact to be established with the test liquid [24, 25]. However, the resulting set-up was cumbersome as the $\mathrm{Pt}$ wire was not easy to insert and stabilize in the reservoir between the metallic zone and the liquid. Additionally, this work should be extended to the production of high-density automated arrays of microfabricated emitter tips for nanoESI applications. This could not be achieved using our previous set-up, which involves a manual step to introduce the metallic wire. A conducting $n$-type doped silicon substrate has been employed here as a support wafer. The ionization 


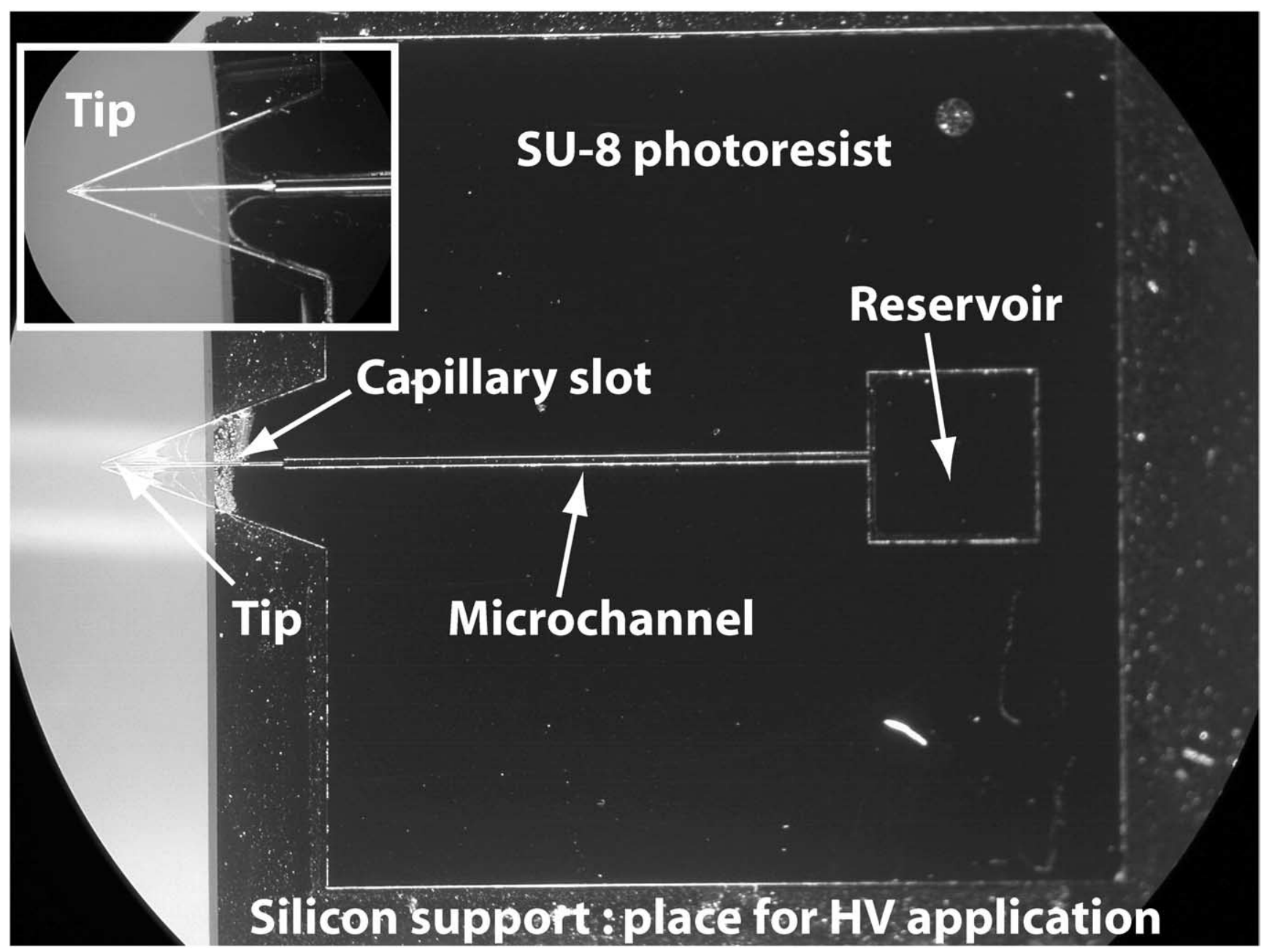

Figure 1. Photograph of a nib emitter tip which is composed of a reservoir feature, a microchannel, a capillary slot, and a point-like structure where electrospraying takes place. The high voltage is applied on the silicon substrate which is in contact with the liquid in the reservoir and in the microchannel.

voltage is applied on the reverse-side of the supporting wafer that is in close contact to the reservoir feature and the microchannel. Consequently, the set-up for the nanoESI-MS tests of the micro-nib emitter tip was greatly simplified without using any metallic wire but rather by directly applying the ionization voltage onto the silicon supporting wafer of the nib structure.

\section{Set-Up Validation Using Standard Peptide Samples}

For validating this new set-up, we started with the same peptide we used in our previous work based on the use of a $\mathrm{Pt}$ wire, i.e., gramicidin $\mathrm{S}$. The peptide sample concentration varied from 1 to $5 \mu \mathrm{M}$ and the ionization voltage was in the range of 1.1 to $1.4 \mathrm{kV}$. The pattern of the resulting mass spectra was consistent with that which had been observed in the original experiments with a very intense peak at $\mathrm{m} / \mathrm{z} 571.5$ corresponding to the $(\mathrm{M}+2 \mathrm{H})^{2+}$ species and a smaller peak at $\mathrm{m} / z 1141.7$ representing the $(\mathrm{M}+\mathrm{H})^{+}$species. Figure 2 (upper panel) presents the mass spectrum obtained for a $1 \mu \mathrm{M}$ gramicidin S sample under a $1.2 \mathrm{kV}$ ionization voltage. It should be noted that by applying the ionization voltage via a Pt wire, we were not able to obtain such spectra with a $1 \mu \mathrm{M}$ sample and voltage values of 1 to $1.2 \mathrm{kV}$ [25]. Instead, the resulting mass spectrum presented two peaks having comparable intensities. This shows that the silicon support set-up is more robust and gives results similar to those obtained using commercial sources under the same operating conditions, whatever the values of the parameters (applied voltage, sample concentration) are. This difference in the mass spectrum pattern using similar test conditions but changing the way to apply the ionization voltage may be accounted for by electrochemistry-linked aspects. First, the conducting microchannel between the reservoir and the nib results in the high voltage being very close to the tip. Second, the surface area between the HV application zone and the test liquid corresponds to the surface of the reservoir bottom and the microchannel, whereas in the former case, this surface was reduced to that of the 


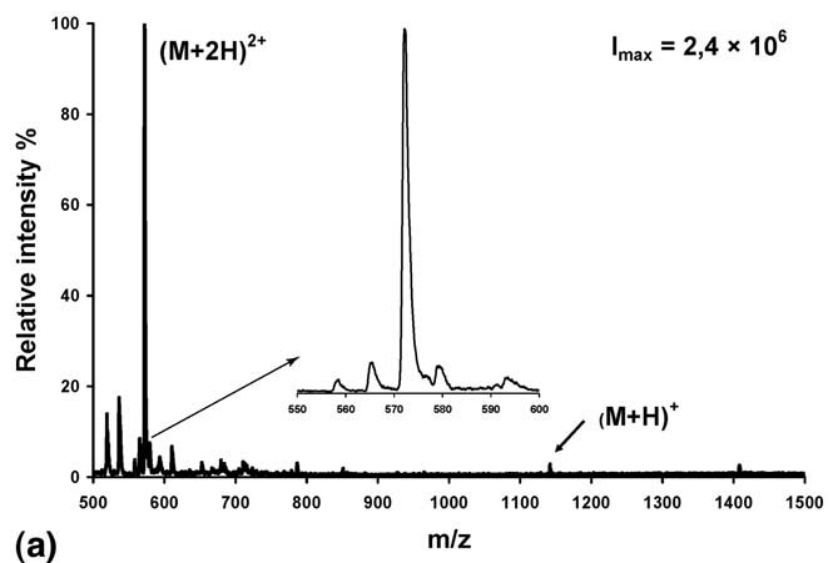

(a)

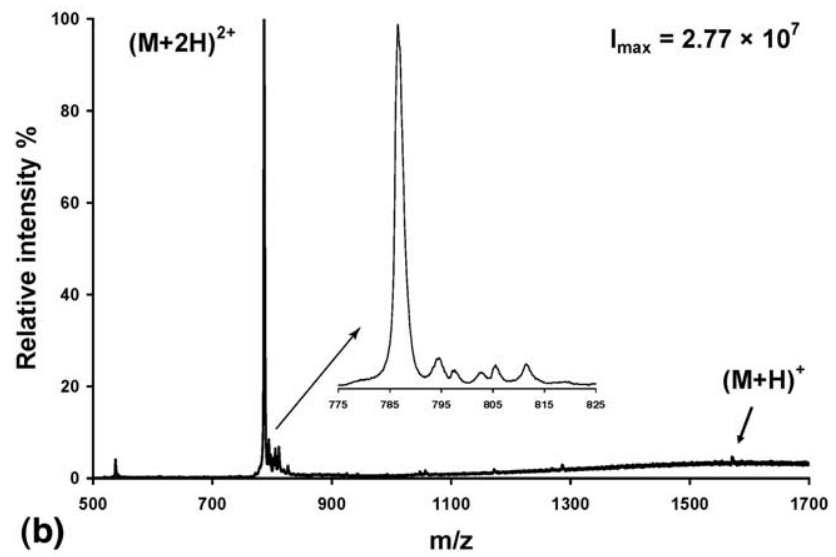

Figure 2. Mass spectra of standard peptide using the micro-nib source with a $8 \mu \mathrm{m}$ slot dimension. Upper panel: mass spectrum averaged on a 2 min acquisition of the signal for a $1 \mu \mathrm{M}$ gramicidin $\mathrm{S}$ sample under a $1.2 \mathrm{kV}$ ionization voltage value; lower panel: mass spectrum averaged on a $6 \mathrm{~min}$ acquisition of the signal for a $1 \mu \mathrm{M}$ Glu-fibrinopeptide B sample under a $1.2 \mathrm{kV}$ ionization voltage value.

Pt wire, i.e., $0.4 \times 10^{-6} \mathrm{~m}^{2}$ (Pt wire) versus $7 \times 10^{-6} \mathrm{~m}^{2}$ ( $\mathrm{Si}$ support). As a result, the current density is much lower in the latter case and the ionization conditions are improved [26, 32].

Subsequently, we tested another standard peptide Glu-fibrinopeptide B that is linear and more representative of proteomic samples contrary to gramicidin S, which is a cyclic and very stable peptide. Glu-fibrinopeptide $B$ is mostly detected at $m / z 786.67$ as a $(\mathrm{M}+2 \mathrm{H})^{2+}$ species and also appears to a lesser extent at $m / z 1570.60$ as a $(\mathrm{M}+\mathrm{H})^{+}$ species. The test conditions were the same as for gramicidin $S$ with voltage values in the range of 1 to $1.4 \mathrm{kV}$ and sample concentrations below $5 \mu \mathrm{M}$. As expected, the doubly-charged species was prominent on the mass spectra together with a slight appearance of the singlycharged ion $(\mathrm{M}+\mathrm{H})^{+}$. Figure 2 (lower panel) represents the mass spectrum obtained with a $1 \mu \mathrm{M}$ Glufibrinopeptide B sample under a $1.2 \mathrm{kV}$ ionization voltage. This mass spectrum is averaged over a $6 \mathrm{~min}$ acquisition period of the signal. We carried out MS/MS experiments on the doubly-charged species under the aforementioned conditions, i.e., $\mathrm{HV}$ of 1 to $1.4 \mathrm{kV}$; concentration $<5 \mu \mathrm{M}$. The $(\mathrm{M}+2 \mathrm{H})^{2+}$ species was specifically isolated (isolation width of 2 Daltons) and fragmented (normalized collision energy parameter of $25 \%$, activation RF frequency factor set at 0.25 ). The observed fragmentation pattern was seen to be the same as with a standard commercial emitter tip, matching peaks having similar relative intensities. The results obtained with standard peptides (gramicidin S, Glufibrinopeptide B) in MS and in MS/MS experiments were consistent with those observed with standard emitter tips. The mass spectra had similar patterns and the analysis conditions were identical in terms of sample concentrations and ionization voltage values.

In the next experiments, we decreased the concentration of the peptide sample. Diluted solutions of Glu-fibrinopeptide B down to $10^{-2} \mu \mathrm{M}$ and $10^{-1} \mu \mathrm{M}$ were prepared and tested in MS using a micro-nib emitter tip in the same conditions as before. These tests were successful without any increase of the voltage supply or any marked decrease in the spectrum intensity. The mass spectrum obtained with the sample at $0.1 \mu \mathrm{M}$ has a good intensity $\left(1.43 \times 10^{6}\right)$ and a good signal-to-noise ratio $(\mathrm{S} / \mathrm{N}=25)$. Analyzing the least concentrated sample at $10^{-2} \mu \mathrm{M}$, the $\mathrm{S} / \mathrm{N}$ and the intensity are both lower, respectively, of around 6 (assessment) and $3.4 \times 10^{5}$ (measured), and there are adducts accompanying the $(\mathrm{M}+2 \mathrm{H})^{2+}$ species start appearing. Lastly, it should be noted that even with a $10^{-2} \mu \mathrm{M}$ sample and a $1.1 \mathrm{kV}$ ionization voltage, the mass spectrum pattern remains unchanged and only presents the $(\mathrm{M}+2 \mathrm{H})^{2+}$ species. Figure 3 presents a mass spectrum obtained with a micro-nib source a Glu-fibrinopeptide B sample at $10^{-2} \mu \mathrm{M}$ under an ionization voltage of $1.1 \mathrm{kV}$; the corresponding TIC signal is presented in the insert of the figure. The micro-nib source had been washed hours before the experiment was performed and no trace of peptide remaining from previous experiments could be de-

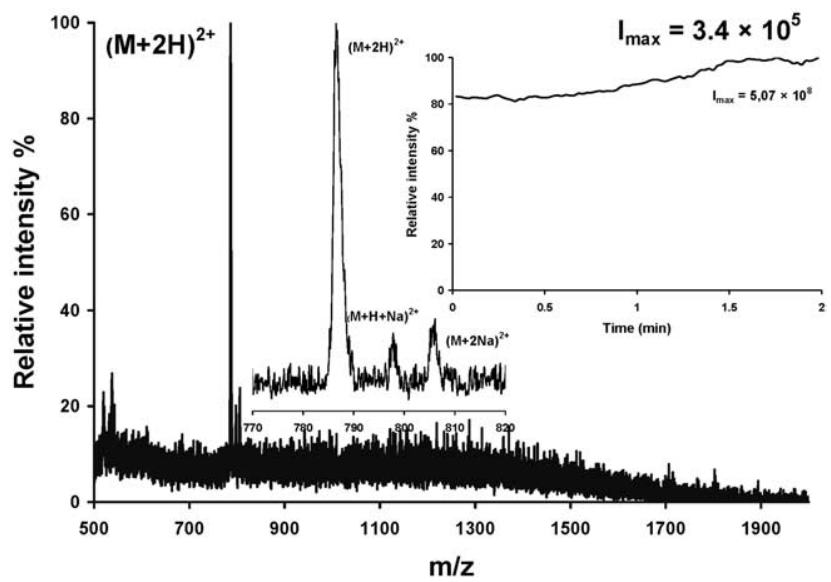

Figure 3. Mass spectrum averaged on a 2 min acquisition of the signal; analysis of a $10^{-2} \mu \mathrm{M}$ Glu Fibrinopeptide B sample under a $1.2 \mathrm{kV}$ ionization voltage value using the micro-nib source with a $8 \mu \mathrm{m}$ slot dimension; inset, corresponding TIC signal recorded for $2 \min (\mathrm{RSD}$ of $7.2 \%$ ). 
Table 1. MS analysis of Glu-fibrinopeptide B samples at various concentrations. Ionization voltage values, maximal intensities measured on TIC signal and di-charged ion signal-to-noise ratio (S/N)

\begin{tabular}{|c|c|c|c|c|c|}
\hline $\begin{array}{l}\text { Sample } \\
\text { concentration }\end{array}$ & HV value & $\mathrm{I}_{\mathrm{TIC}}$ & $\mathrm{I}_{\mathrm{MS}}$ & $\mathrm{S} / \mathrm{N}$ & $\mathrm{I}_{\text {Mono }} / \mathrm{I}_{\mathrm{Di}}$ \\
\hline $5 \mu \mathrm{M}$ & $1.2 \mathrm{kV}$ & $1.16 \times 10^{10}$ & $1.18 \times 10^{8}$ & 250 & $\mathrm{I}_{\mathrm{Mono}} / \mathrm{I}_{\mathrm{Di}}=4.3 \times 10^{-2}$ \\
\hline $1 \mu \mathrm{M}$ & $1.2 \mathrm{kV}$ & $9.7 \times 10^{9}$ & $2.3 \times 10^{7}$ & 167 & $\mathrm{I}_{\mathrm{Mono}} / \mathrm{I}_{\mathrm{Di}}=1.5 \times 10^{-2}$ \\
\hline $0.1 \mu \mathrm{M}$ & $1.1 \mathrm{kV}$ & $1.19 \times 10^{9}$ & $1.43 \times 10^{6}$ & 25 & $\mathrm{I}_{\mathrm{Mono}} / \mathrm{I}_{\mathrm{Di}}=1.46 \times 10^{-2}$ \\
\hline $0.01 \mu \mathrm{M}$ & $1.1 \mathrm{kV}$ & $5.07 \times 10^{8}$ & $3.4 \times 10^{5}$ & 6 & $\mathrm{No}(\mathrm{M}+\mathrm{H})^{+}$ \\
\hline
\end{tabular}

tected before this test was carried out. Figure 3 shows also that the sodium and potassium salt adducts on the doubly-charged species remain limited, even at this concentration; this emphasizes the advantage of the chosen materials SU-8 and silicon compared with borosilicate glass or fused-silica, which are conventionally used for classical tips. Table 1 summarizes the results in terms of TIC maximum intensity, mass spectrum maximum intensity, as well as an estimated value of the signal-to noise ratio obtained for a series of tests using samples of Glu-fibrinopeptide B samples at different concentrations. Using these data, we trace a log-log plot for the mass spectrum intensity as a function of the sample concentration, which shows a linear correlation between the mass spectrum intensity and the sample concentration with a 0.97 slope value and a very good correlation coefficient value $\left(R^{2}=0.98\right)$. This is in agreement with the model proposed by Tang and Kebarle for a linear dependence of the analyte signal intensity as a function of its concentration with a unitary slope value as long as the concentration is lower than $10 \mu \mathrm{M}$ [33].

These data allowed us to discuss the major drawback of the open design of the nib device, i.e., sample duration. A volume of one microliter of sample lasts for $\sim 5 \mathrm{mn}$, which is indeed lower than a classical nano-ESI tip. However, electrospray ionization is a concentration-dependant process and the signal that is detected by the mass spectrometer is not affected by the flow-rate value [34]. Indeed, ESI and nano-ESI have almost the same sensitivity in spite of a $10^{3}$-fold increase in the sample consumption in the case of ESI versus nanoESI. Thus, the higher sensitivity observed on the nib device cannot be accounted for by a higher flow-rate value. In-source evaporation of the solvent attributable to the current open topology of the micro-nib sources leading to an in-reservoir concentration of the peptide solution is also not a concern. It should be noted that the spectrum presented in Figure 3 was obtained immediately after loading the $10^{-2} \mu \mathrm{M}$ and that the observed intensity was constant during the all the experiments up to $10 \mathrm{~min}$. In the future, we plan to include a cover plate on the reservoir. Thus, the contact surface area between the liquid sample and the air could be decreased to give reduced evaporation of the sample in the source without modifying the open design.

\section{Conclusions}

We have presented a microfabricated ionization tip with the geometry and functioning of a fountain pen that works under true nanoESI conditions. The MS set-up reported here offers the possibility of a direct application of the ionization voltage onto the silicon support without using a metallic wire. It was seen that the ionization conditions were much gentler using this set-up, which was validated using peptide concentration down to $10^{-2} \mu \mathrm{M}$. Analysis conditions in terms of voltage values and sample concentrations were comparable to those of standard nanospray emitter tips. Furthermore, the nib tip may be used for quantitative work as its response in nanoESI exhibits a linear variation as a function of peptide concentration in the range of $10 \mu \mathrm{M}$ to $10 \mathrm{nM}$. Nib tips will be of great interest for proteomics studies as they apparently exhibit enhanced analysis capabilities in terms of sensitivity and species detection range.

\section{Acknowledgments}

The authors thank the GenHomme network (BioChipLab consortium). The Mass Spectrometry facilities used for this study are funded by the European community (FEDER), the Région NordPas de Calais (France), the CNRS, and the Universite des Sciences et Technologies de Lille. The authors also thank Christophe Boyaval of IEMN for the Scanning Electron Microscopy (SEM) images.

\section{References}

1. Patterson, S. D.; Aebersold, R. H. Proteomics: The first decade and beyond. Nat. Genet. 2003, 33, 311-323.

2. Wilm, M.; Mann, M. Analytical properties of the nanoelectrospray ion source. Anal. Chem. 1996, 68, 1-8.

3. Aebersold, R. A mass spectrometric journey into protein and proteome research. J. Am. Soc. Mass Spectrom. 2003, 14, 685-695.

4. Feng, B.; Smith, R. D. A simple nanoelectrospray arrangement with controllable flow rate for mass analysis of submicroliter protein samples. J. Am. Soc. Mass Spectrom. 2000, 11, 94-99.

5. Nilsson, S.; Svedberg, M.; Pettersson, J.; Bjoerefors, F.; Markides, K.; Nyholm, L. Evaluations of the stability of sheathless electrospray ionization mass spectrometry emitters using electrochemical techniques. Anal. Chem. 2001, 73, 4607-4616.

6. Valaskovic, G. A.; Kelleher, N. L.; Little, D. P.; Aaserud, D. J.; McLafferty, F. W. Attomole-sensitivity electrospray source for large-molecule mass spectrometry. Anal. Chem. 1995, 67, 3802-3805.

7. Valaskovic, G. A.; McLafferty, F. W. Long-lived metallized tips for nanoliter electrospray mass spectrometry. J. Am. Soc. Mass Spectrom. 1996, 7, 1270-1272.

8. Barnidge, D. R.; Nilsson, S.; Markides, K. E.; Rapp, H.; Hjort, K. Metallized sheathless electrospray emitters for use in capillary electrophoresis orthogonal time-of-flight mass spectrometry. Rapid Commun. Mass Spectrom. 1999, 13, 994-1002.

9. Goss, C. A.; Charych, D. H.; Majda, M. Application of (3-mercaptopropyl)trimethoxysilane as a molecular adhesive in the fabrication of 
vapor-deposited gold electrodes on glass substrates. Anal. Chem. 1991, 63, 85-88.

10. Kriger, M. S.; Cook, K. D.; Ramsey, R. S. Durable gold-coated fused silica capillaries for use in electrospray mass spectrometry. Anal. Chem. 1995, 67, 385-389.

11. Lin, L.; Pisano, A. P. Silicon-processed microneedles. IEEE J. Microelectromech. Syst. 1999, 8, 78-84.

12. Licklider, L.; Wang, X.-Q.; Desai, A.; Tai, Y.-C.; Lee, T. D. A micromachined chip-based electrospray source for mass spectrometry. Anal. Chem. 2000, 72, 367-375.

13. Schultz, G. A.; Corso, T. N.; Prosser, S. J.; Zhang, S. A fully integrated monolithic microchip electrospray device for mass spectrometry. Anal. Chem. 2000, 72, 4058-4063.

14. Sjödahl, J.; Melin, J.; Griss, P.; Emmer, A.; Stemme, G.; Roeraade, J. Characterization of micromachined hollow tips for two-dimensional nanoelectrospray mass spectrometry. Rapid Commun. Mass Spectrom. 2003, 17, 337-341.

15. Griss, P.; Melin, J.; Sjödahl, J.; Roeraade, J.; Stemme, G. Development of micromachined hollow tips for protein analysis based on nanoelectrospray ionization mass spectrometry. J. Micromech. Microeng. 2002, 12, 682-687.

16. Tang, K.; Lin, Y.; Matson, D. W.; Kim, T.; Smith, R. D. Generation of multiple electrosprays using microfabricated emitter arrays for improved mass spectrometric sensitivity. Anal. Chem. 2001, 73, 1658-1163.

17. Schilling, M.; Nigge, W.; Rudzinski, A.; Neyer, A.; Hergenroeder, R. A new on-chip ESI nozzle for coupling of MS with microfluidic devices. Lab on a Chip 2004, 4, 220-224.

18. Yuan, C.-H.; Shiea, J. Sequential electrospray analysis using sharp-tip channels fabricated on a plastic chip. Anal. Chem. 2001, 73, 1080-1083.

19. Kim, J. S.; Knapp, D. R. Microfabricated PDMS multichannel emitter for electrospray ionization mass spectrometry. J. Am. Soc. Mass Spectrom. 2001, 12, 463-469.

20. Svedberg, M.; Veszelei, M.; Axelsson, J.; Vangbo, M.; Nikolajeff, F. Poly(dimethylsiloxane) microchip: Microchannel with integrated open electrospray tip. Lab on a Chip 2004, 4, 322-327.

21. Gobry, V.; Van Oostrum, J.; Martinelli, M.; Rohner, T. C.; Reymond, F.; Rossier, J. S.; Girault, H. H. Microfabricated polymer injector for direct mass spectrometry coupling. Proteomics 2002, 2, 405-412.
22. Rossier, J. S.; Youhnovski, N.; Lion, N.; Damoc, E.; Becker, S.; Reymond, F.; Girault, H. H.; Przybylski, M. Thin-chip microspray system for high-performance Fourier-transform ion-cyclotron resonance mass spectrometry of biopolymers. Angew. Chem. Int. Ed. 2003, 42, 53-58

23. Kameoka, J.; Orth, R.; Ilic, B.; Czaplewski, D.; Wachs, T.; Craighead, H. G. An electrospray ionization source for integration with microfluidics. Anal. Chem. 2002, 74, 5897-5901.

24. Le Gac, S.; Arscott, S.; Cren-Olive, C.; Rolando, C. Two-dimensional microfabricated sources for nanoelectrospray. J. Mass Spectrom. 2003, 38, 1259-1264.

25. Le Gac, S.; Arscott, S.; Rolando, C. A planar microfabricated nanoelectrospray emitter tip based on a capillary slot. Electrophoresis 2003, 24, 3640-3647.

26. Van Berkel, G. J.; Asano, K. G.; Schnier, P. D. Electrochemical processes in a wire-in-a-capillary bulk-loaded, nano-electrospray emitter. J. Am. Soc. Mass Spectrom. 2001, 12, 853-862.

27. Kertesz, V.; Van Berkel, G. J. Minimizing analyte electrolysis in an electrospray emitter. J. Mass Spectrom. 2001, 36, 204-210.

28. Arscott, S.; Le Gac, S.; Druon, C.; Tabourier, P.; Rolando, C. A micro-nib nanoelectrospray source for mass spectrometry. Sens. Actuators B 2004, 98, 140-147.

29. Le Gac, S.; Cren-Olive, C.; Rolando, C.; Arscott, S. A novel nib-like design for microfabricated nanospray tips. J. Am. Soc. Mass Spectrom. 2004, 15, 409-412.

30. Arscott, S.; Le Gac, S.; Druon, C.; Tabourier, P.; Rolando, C. A planar on-chip micro-nib interface for nano-ESI-MS microfluidic applications. J. Micromech. Microeng. 2004, 14, 310-316.

31. Brinkmann, M.; Blossey, R.; Arscott, S.; Druon, C.; Tabourier, P.; Le Gac, S.; Rolando, C. Microfluidic design rules for capillary slot-based electrospray sources. Appl. Phys. Lett. 2004, 85, 2140-2142.

32. Van Berkel, G. J.; Zhou, F. Characterization of an electrospray ion source as a controlled-current electrolytic cell. Anal. Chem. 1995, 67, 2916-2923.

33. Tang, L.; Kebarle, P. Dependence of ion intensity in electrospray mass spectrometry on the concentration of the analytes in the electrosprayed solution. Anal. Chem. 1993, 65, 3654-3668.

34. Ikonomou, M. G.; Blades, A. T.; Kebarle, P. Electrospray-ion spray: A comparison of mechanisms and performance. Anal. Chem. 1991, 63, 1989-1998. 